\title{
Manejo inmediato de intrusión dental y lesión en tejidos blandos: Reporte de caso
}

\author{
Prompt treatment of dental intrusion and in soft tissue injuries. Case Report \\ Eder Hernández-Hernández ${ }^{1 a}$, Laura Vargas Servin ${ }^{1 b}$, Carlo Medina Solís ${ }^{1 b}$, Carlos Varela Ibañez ${ }^{1, c}$, Maria \\ Anton Baños ${ }^{1 a}$
}

\section{RESUMEN}

Los traumatismos dentales son eventos que suceden frecuentemente. Los datos estadísticos revelan cifras considerables en cuanto a la incidencia y prevalencia de estos accidentes. La intrusión dental es de las más comunes durante la infancia y su tratamiento depende de la severidad del caso. Junto con los traumatismos dentales se presentan lesiones en tejidos blandos, las cuales deben ser tratadas del modo más conservador posible a fin de reducir las posibles secuelas. Presentación del caso: En el reporte se describe a una paciente femenina de 2 años 8 meses de edad que acude al área de urgencias del Hospital del Niño DIF Hidalgo por presentar "caída de las escaleras”. Acude en primera instancia a otro hospital de donde fue remitida 24 horas después. A la exploración clínica bucal se observa intrusión de dientes 51 y 61. Clínica y radiográficamente apreciamos fractura de tabla alveolar vestibular. Presenta lesión lacerante en encía a nivel de dichos órganos y en el labio inferior. Se realiza lavado de la herida, se extraen los dientes afectados y se suturan las heridas. Se realizó seguimiento a la paciente durante 3 meses observando correcta cicatrización de las heridas. Conclusiones: La paciente presenta una evolución correcta. El manejo oportuno inmediato en este tipo de lesiones es esencial a fin de disminuir las posibles secuelas.

PALABRAS CLAVE: Traumatismos dentales, preescolares, lesiones bucales.

\section{SUMMARY}

Dental trauma are events that happen often. Stadistic revels high numbers about incidence and prevalence of these accidents. Dental intrusion is one of the most common injuries among childhood and the treatment de-

\footnotetext{
${ }^{1}$ Instituto de Ciencias de la Salud, Universidad Autónoma del Estado de Hidalgo. Pachuca, Hidalgo, México.

a CD, Especialista en Odontopediatría.

b CD, Magister.

${ }^{c}$ CD, Especialista en Cirugía MaxiloFacial.
} 
pends of the severity of the case. Dental trauma presents also soft tissue injuries, which must be treated in the most conservative way to reduce possible sequels. Case report: In this paper is described a female patient of two years eight month old which is carried by her mother to the urgency room of the Hospital del Niño DIF Pachuca because she suffered a fell from stairs. She went first to other Hospital and is dispatched to specialized care 24 hours later. At clinical examination we see intrusion of teeth 51 y 61 and lacerating injury of gingiva and lower lip. Clinical and radiographic appreciate fracture of the vestibular alveolar table. The wound is washed, affected tooth are extracted and sutured the injury. We conducted follow-up of the patient for 3 months observing proper healing of wounds. Conclusions: The evolution of the patient was satisfactory. The immediate prompt handling these types of injuries is essential in order to reduce the potential consequences

\section{KEY WORDS: Dental trauma, buccal injuries, children}

\section{INTRODUCCIÓN}

Entre las principales urgencias estomatológicas se encuentran los traumatismos dentoalveolares, pues son situaciones de dolor, molestia y alteración funcional repentina al paciente, así como angustia a sus familiares; que hacen que quien lo padezca acuda al odontólogo. Para Andreasen y Ravn, cerca de un 30\% de niños menores de 7 años han sufrido un traumatismo dental en algún momento de su vida. Entre estos las intrusiones dentales ocupan entre el 8 y el 22\% de las luxaciones reportadas (1).

Los traumatismos dentoalveolares son lesiones que afectan a los dientes y al hueso que lo sostiene. Estos provocan una disminución de las capacidades de masticación y dicción, así como problemas estéticos que pueden producir trastornos psicológicos, sociales y terapéuticos.Además pueden ir acompañados de lesiones en los tejidos blandos con hemorragia e inflamación, que pueden impresionar a los familiares o acompañantes del paciente traumatizado (2). Los factores más comunes que resultan en traumatismos dentales en los niños son las caídas cuando aprenden a caminar y a correr, la práctica de deportes y los accidentes automovilísticos, retraso mental, enfermedades convulsivas, sin olvidar al niño maltratado y las situaciones de abuso (1-3).

En niños de edad preescolar los traumatismos orales son los segundos accidentes físicos más comunes y entre éstos los traumatismos dentales son los más frecuentes, seguidos de las lesiones de tejidos extraorales y por último, lesiones del hueso alveolar (1). Hoy en día los traumatismos dentales son la segunda causa de atención odontopediátrica después de la caries (4).
La evaluación de los traumas en la dentición primaria es muy relevante no sólo por la presencia de secuelas en esa dentición, sino también porque permite la identificación de alteraciones posibles de desarrollo de la dentición permanente, por lo cual se requiere de una exploración inmediata y controles a largo plazo, para evitar producir estas complicaciones y patologías pasado un tiempo de haberlos padecido $(5,6)$. Otros factores a considerar es la cercanía del ápice del diente afectado con el germen del diente permanente, la integridad de la tabla alveolar, la presencia de abscesos $(7,8)$.

En intrusiones dentales el tratamiento recomendado varía dependiendo la severidad de la lesión. Von Hartz (6) los clasifica de la siguiente manera:

Grado I. Intrusión parcial leve: donde se observa más del 50\% de la corona clínicamente.

Grado II. Intrusión parcial moderada: donde se observa menos del 50\% de la corona.

Grado III. Intrusión severa: donde no se observa porción alguna de la corona.

El tratamiento de elección será tan conservador como sea posible, pero tan radical como sea necesario. Según la severidad de la lesión y previo análisis de las características individuales del paciente se puede optar por la reerupción espontánea o la odontectomía $(1,2)$. En tejidos blandos el tratamiento debe ser enfocado a la preservación de la función y a conservar la estética limitando las secuelas del daño $(3,4)$. Por desgracia, la gente en general no está bien informada sobre los riesgos del trauma en la dentición decidua y permanente y lo que se puede hacer para evitarlos (9).

Se elige este caso para enfatizar la importancia del manejo inmediato de los traumatismos dentoalveola- 
res, ya que este es crítico para el mejor pronóstico en este tipo de eventos.

\section{REPORTE DEL CASO}

Se trata de paciente femenina de 2 años 8 meses de edad que acude acompañada de su madre al área de urgencias del Hospital del niño DIF Hidalgo el día 3 de abril de 2013, quien fue remitida de otro hospital para su atención especializada. La atención recibida en dicho nosocomio incluyó radiografía lateral de cráneo y posteroanterior; con diagnóstico de avulsión de órganos dentales (Figura 1). Al interrogatorio la madre no refiere antecedentes clínicos de importancia para el caso. La madre manifiesta que la paciente cayó de las escaleras de su casa, rodando 4 escalones, y como consecuencia sufre heridas en boca 24 horas antes de su ingreso al servicio.

Es recibida en el servicio de urgencias y se solicita interconsulta al área de odontopediatría para valoración y tratamiento.

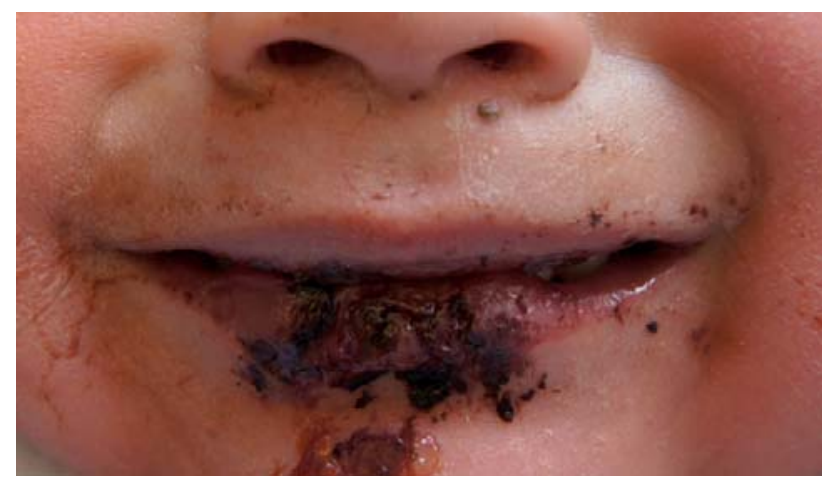

Figura 1. Paciente niña de 2 años 8 meses de edad con golpe en labio inferior.
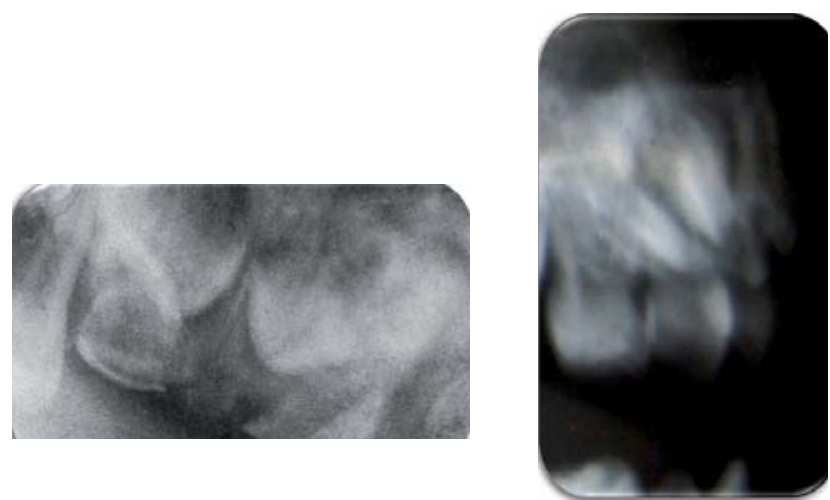

Figura 2. Radiografías donde se observa la intrusión dental.
Al analizar las radiografías se observó que los órganos dentales (OD) 51 y 61 no presentaban avulsión sino intrusión completa (Grado III de Von Hartz), además de fractura de la tabla vestibular (Figura 2).

La paciente se presentó irritable y poco cooperadora con llanto obstinado y compensatorio. Previa firma de consentimiento informado por la madre, se realiza la exploración física usando la técnica de restricción física; en la valoración se encuentra a la paciente consciente, activa, reactiva, normocránea con coloración adecuada de piel y mucosas; presentaba restos hemáticos en boca y una lesión lacerante de aproximadamente de $4 \mathrm{~cm}$ en labio inferior con restos de escara. Intraoralmente se identificó cavidad oral en malas condiciones de higiene con restos hemáticos, dentición temporal en proceso de erupción. Ausencia clínica de OD 51 y 61 con lesión lacerante en encía en sector anterosuperior.

Para complementar el diagnostico se toma radiografía periapical donde se observa intrusión completa de OD 51 y 61.

Para la planeación del tratamiento se siguen las recomendaciones de la IADT (International Asociation of Dental Traumatology) (10) y se decide realizar odontectomía de OD 51 y 61 por el riesgo de afectación a los dientes permanentes (Figuras 3 y 4) El procedimiento clínico se inicio con la selección de lídocaina con epinefrina como anestésico local debido a su bajo riesgo de toxicidad y su adecuada latencia.

Se anestesia usando $36 \mathrm{mg}$ de lidocaína con epinefrina 1:100 000 por vía infiltrativa local. Se realizó lavado de heridas con yodopovidona al 10\% por su eficacia contra microorganismos grampositivos y gramnegativos; y se continuó con la odontectomía usando un elevador recto. Durante la odontectomia se retira la tabla vestibular fracturada. Una vez finalizado el procedimiento se colocaron puntos de sutura simples con vycril 3-0. La herida en el labio igualmente se lavó con yodopovidona al 10\%. Se retiraron además, los bordes necróticos con tijeras para encía.

Se realizó lavado mecánico riguroso a fin de eliminar la escara y conseguir una superficie cruenta para promover la adecuada cicatrización. Al ser el labio una estructura muscular móvil precisaba ser cerrada por planos: superficial y profundo e igualmente se 
utiliza vycril 3-0 (Figuras 5 y 6).

Posterior al procedimiento clínico se prescribió terapia farmacológica con base en analgesia y antibioticoterapia. Para el primero, se indica ibuprofeno a 8 $\mathrm{mg} / \mathrm{kg} /$ dosis y para el segundo amoxicilina con acido clavulánico a 50 mg/kg/día (10).

Una vez terminado el procedimiento se dieron indicaciones de cuidado por escrito a la madre, las cuales incluyeron: cuidado de zona anestesiada, higiene oral estricta con la utilización de cepillo dental de cerdas blandas y técnica de cepillado de Bass modificada, evitar llanto de la niña, dieta blanda, lavado extraoral de zona afectada con agua limpia y una gasa para evitar formación de escara. Se dió cita ocho días después para revisión.
Ocho dias despues, la paciente acude en compañía de la madre. A la exploración fisica se observa que no se siguieron las indicaciones prescritas. La herida se presenta con dehisencia e inadecuado proceso de cicatrizacion, en malas condiciones de higiene y con una formacion de escara que cubre la superficie completa de la lesión.

En particular, en este caso ya no fue conveniente manipular por segunda ocasión la lesión debido al tiempo trascurrido. Por tanto se le indica reforzar la higiene bucodental y uso de crema fitoestimuladora 3 veces al día. Se cita 15 días después para nueva revisión.

La madre se presenta junto con la paciente un mes después y en esta ocasión se observa franca mejoria en el proceso de cicatrización (Figura 7).

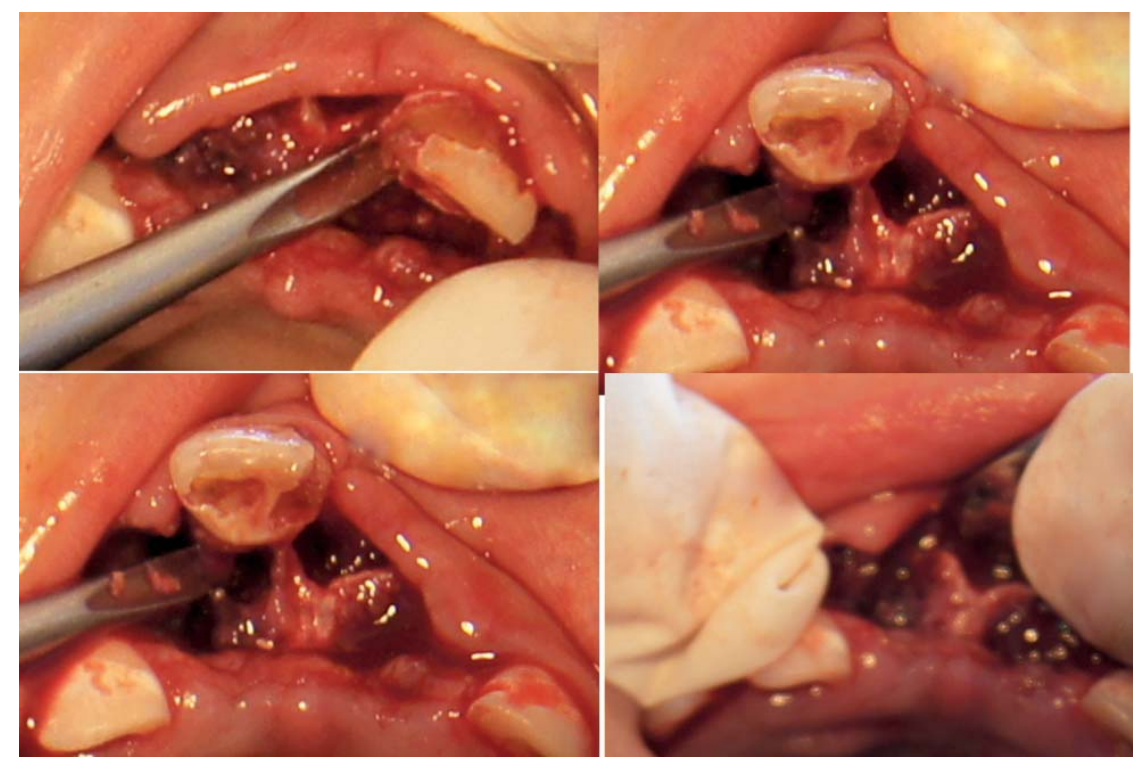

Figura 3. Odontectomía de OD 51

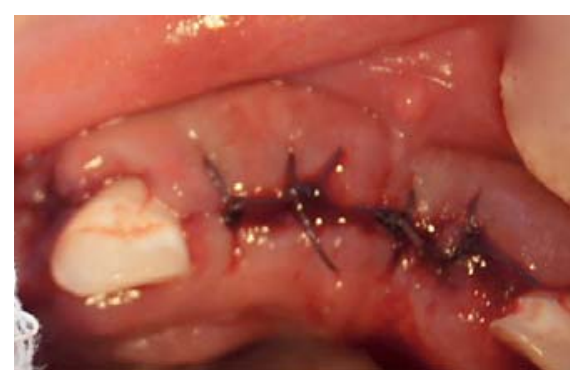

Figura 4. Odontectomía de OD 61

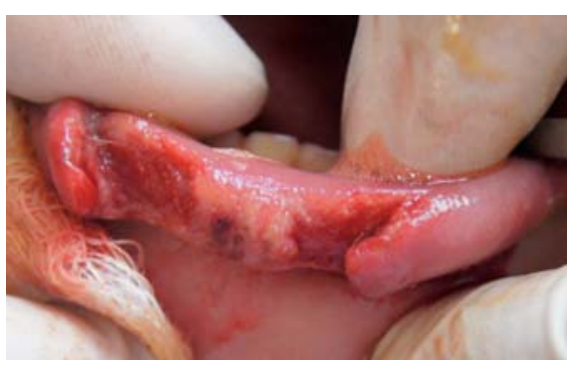

Figura 5. Lesión de labio inferior. 


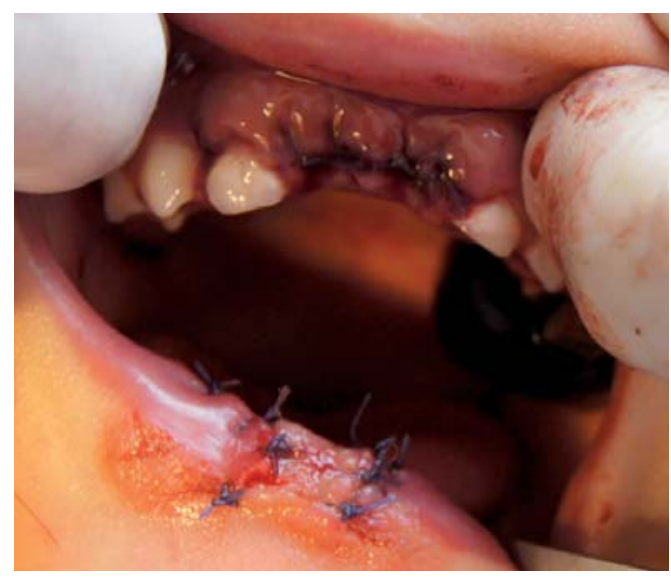

Figura 6. Cierre del labio por plano profundo utilizando vycril 3-0.

\section{DISCUSIÓN}

Se ha reportado en la literatura científica que el pico de incidencia para traumatismos dentoalveolares en dentición temporal es entre los 2 y 3 años de edad, siendo los más afectados los incisivos superiores; lo cual coincide con las características epidemiológicas del presente caso clínico.

Con frecuencia, los pacientes que acuden por este tipo de urgencias no han tenido contacto previo con un dentista. Estos pacientes se presentan con dolor, asustados y son poco cooperadores y es necesario recurrir a la restricción física. Debido a la urgencia de estos procedimientos no es posible realizar técnicas de modelado de conducta con un enfoque más conservador. Lo que causa traumas y fobias a los tratamientos dentales por parte de los pacientes.

Para el tratamiento de estos pacientes, estudios previos sugieren adoptar un enfoque conservador para resolver estos casos $(2,4)$. Se recomienda esperar a la reerupción espontánea de los órganos dentales siempre que sea posible. Sin embargo, en este caso en particular se optó por un tratamiento más radical debido a la fractura de la tabla alveolar vestibular, como lo recomiendan algunos autores $(5,7)$. Los dientes permanentes podrían presentar secuelas como: (retraso en la erupción, anomalías estructurales e hipopla-

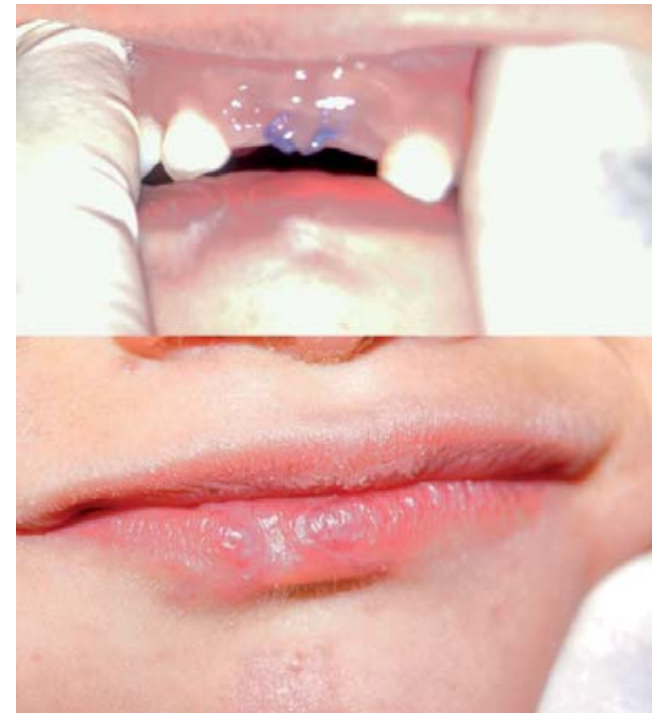

Figura 7. Paciente presenta franca mejoria en el proceso de cicatrización

sia). Así mismo, se buscó evitar el riesgo de secuelas en los mismos O.D. intruídos tales como: infección, anquilosis, obliteración del canal pulpar, dilaceración de las raíces, necrosis y formación de quistes. Además de evitar afectación del germen del diente permanente adyacente, anquilosis de los dientes deciduos, así como infección y/o necrosis de la zona lesionada $(7,11)$.

Realizar una extracción a edades tempranas puede tener secuelas en cuanto a la función masticatoria, fonación, oclusión y formación de hábitos perniciosos, como proyección lingual. Para ello se debe continuar con la rehabilitación protésica a fin de restablecer la función y la estética $(7,8)$.

El uso de vycril como material de sutura es adecuado, ya que éste es un material reabsorbible con adecuada fuerza tensil para sostener los tejidos y elimina la necesidad del retiro posterior de puntos (9).

El manejo en los tejidos blandos se recomienda que el lavado sea inmediatamente después del evento traumático a fin de eliminar partículas extrañas $(9,12)$. En el caso que se presenta no fue posible ya que la paciente es llevada a atención odontopediátrica 24 horas después del evento. Por otro lado, aunque en la literatura no se reporta el beneficio usando la crema fitoestimuladora, este se indica para condicio- 
nar a la madre a realizar el lavado de la herida al ajustarse a un horario indicado.

Es importante además, ser muy claros al dar las indicaciones a los padres de familia ya que su cooperación es vital para la evolución satisfactoria (9). La madre de la paciente no llevo a cabo las instrucciones como fueron prescritas, lo que dio como resultado un proceso de cicatrización inadecuado y dejando la marca de cicatriz en el labio inferior en su porción derecha de aproximadamente $2 \mathrm{~cm}$, sin embargo la paciente conserva la capacidad sensomotriz de la zona.

Aunque se buscó mantener un seguimiento de control de esta paciente para rehabilitación protésica y vigilancia de los dientes permanentes la madre deja de acudir a consulta.

\section{CONCLUSIONES}

Los traumatismos dentoalveolares son una problemática compleja ya que implica diversos aspectos funcionales y estéticos a considerar.

Al enfrentar este tipo de padecimientos es importante realizar un diagnóstico adecuado y considerar las características específicas de cada paciente. No siempre es posible encontrar las condiciones ideales para realizar el tratamiento, así que nuestros objetivos deben dirigirse a conservar la función y la estética.

El interrogatorio y el examen clínico, así como, la interpretación radiográfica deben ser minuciosos a fin de no pasar por alto detalles determinantes en el diagnóstico y plan de tratamiento.

La cooperación y el entendimiento de los padres son fundamentales para lograr resultados óptimos, por lo que es necesario comprometerlos a realizar adecuadamente el cuidado y la limpieza de las heridas.

Finalmente es importante resaltar que el manejo inmediato de un traumatismo dentoalveolar en pacientes pediátricos es crítico para reducir el riesgo de que se presenten secuelas negativas, por lo que se recomienda realizar un análisis clínico exhaustivo a fin de escoger la terapéutica apropiada dependiendo de cada caso.

\section{Correspondencia:}

Carlo Eduardo Medina Solís

correo electrónico: cemedinas@yahoo.com

\section{REFERENCIAS BIBLIOGRAFICAS}

1. Andreasen JO. Etiology and pathogenesis of traumatic dental injuries.A clinical study of 1,298 cases. Scand J Dent Res. 1970;78:329342.

2. Hirata R, Kaiharaet Y, Suzuki J, Kozai K. Management of intruded primary teeth after traumatic injuries. Pediatric Dental Journal. 2011;21:94-100.

3. Flores MT, Andreasen JO, Bakland LK. Guidelines for the evaluation and management of traumatic dental injuries. Dent Traumatol. 2011;17:49-52.

4. Zhang Y, Zhu Y, Su W, Zhou Z, Jin Y, Wang X. A retrospective study of pediatric traumatic dental injuries in Xi'an China. Dent Traumatol. 2014;30:211-5.

5. Holan G, Needleman HL. Premature loss of primary anterior teeth due to trauma - potential short- and long-term sequelae. Dent Traumatol. 2014;30:100-6.

6. Al-Jundi SH. Type of treatment, prognosis, and estimation of time spent to manage dental trauma in late presentation cases at a dental teaching hospital: a longitudinal and retrospective study. Dent Traumatol. 2004;20:1-3

7. Assuncao LR, Ferelle A, Iwakura ML, et al. Effects on permanent teeth after luxation injuries to the primary predecessors: a study in children assisted at an emergency service. Dent Traumatol. 2009;25:16570 .

8. Gondim JO, Moreira Neto JS. Evaluation of intruded primary incisors. Dent Traumatol. 2005;21:131-3.

9. Sae-Lim V, Chulaluk K, Lim LP. Patient and parental awareness of the importance of the importance of immediate management of traumatized teeth. Endod Dent Traumatol. 1999;15:37-4.

10. International Association of Dental Traumatology (IADT). Copenhagen: University Hospital of Copenhagen; 2013 (Citado el 2 de noviembre 2013) Disponible en: http://www.iadt-dentaltrauma.org/

11. Kramer PF, Zembruski C, Ferreira SH, Feldens CA. Traumatic dental injuries in Brazilian preschool children. Dent Traumatol 2003;19:299-303.

12. Skaare AB, MasengAas AL, Wang NJ. Enamel defects in permanent in cisors after trauma to primary predecessors: inter-observer agreement based on photographs. Dent Traumatol. 2013;29:79-83.

Recibido : 07/01/2015

Aceptado: 30/05/2015 